Reflexiones contables (Cúcuta)

8

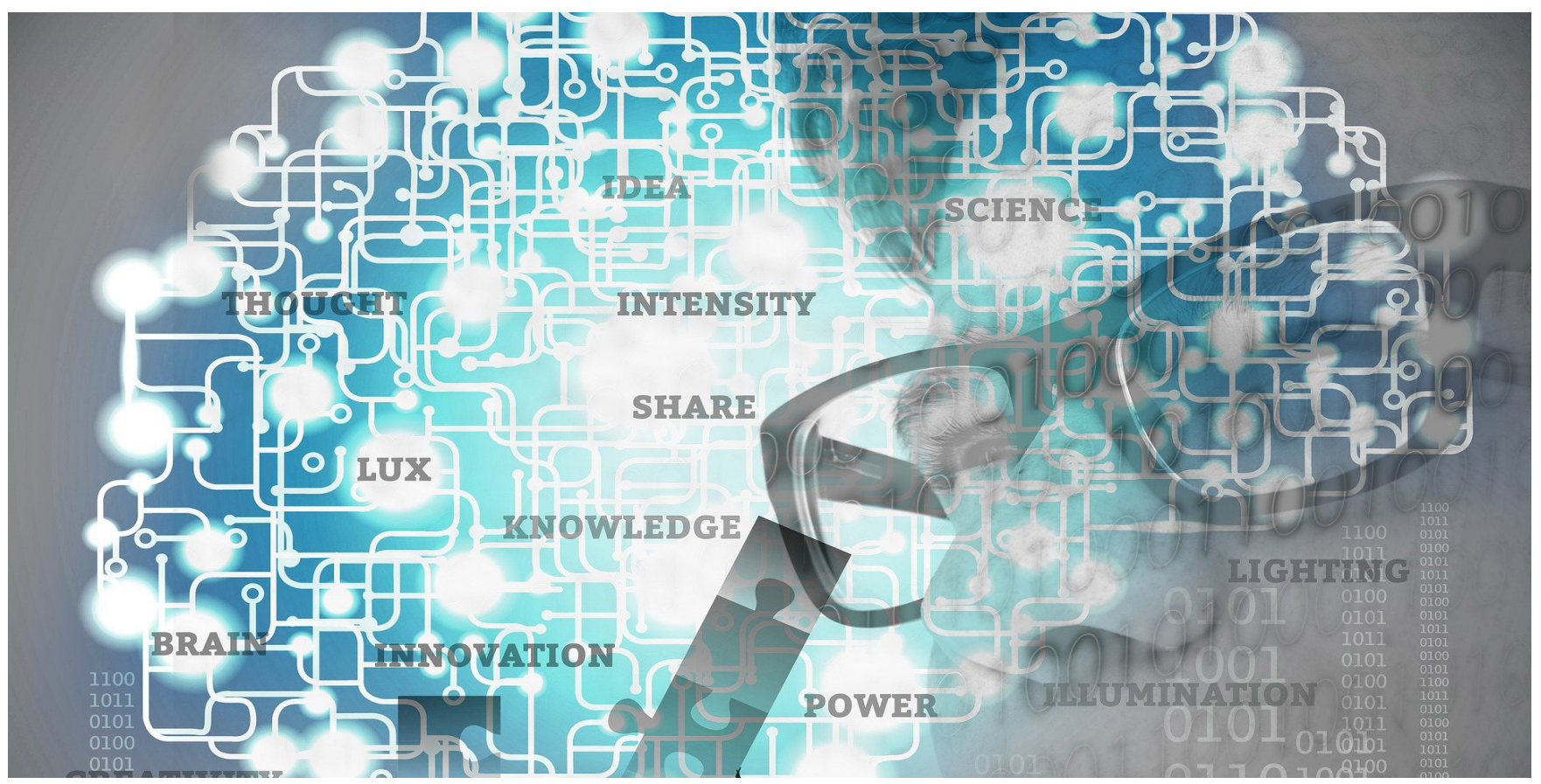

\title{
Tendencias actuales de la industria 4.0
}

\section{Current Industry 4.0 Trends}

Lilia Concepción Ríos-Ramírez

Estudiante de Ingeniería Industrial y de Sistemas

al143377@alumnos.uacj.mx, Universidad Autónoma de

Ciudad de Juárez, Ciudad de Juárez. México

Luis Pérez-Domínguez

Doctor en Ciencias de la Ingeniería

luis.dominguez@uacj.mx,

Universidad Autónoma de Ciudad de Juárez, Ciudad de
Iván Juan Carlos Pérez-Olguin

Doctor en Ciencias e Ingeniería Industrial

ivan.perez@uacj.mx,

Universidad Autónoma de Ciudad de Juárez, Ciudad de Juárez. 
Cómo citar: Ríos-Ramírez, L.C., Pérez-Domínguez, L. \& Pérez-Olguin, I.J.C. (2019). Tendencias actuales de la industria 4.0. Reflexiones contables (Cúcuta), 2 (2), 8-22.

\section{Resumen}

El presente artículo aborda una exploración literaria de la industria 4.0 en los años más recientes. La industria 4.0 es la actual revolución industrial, la cual consiste en la digitalización de los procesos industriales con ayuda de las tecnologías de la información (TI) y la inteligencia artificial (IA). Esto implica combinar las técnicas avanzadas de la producción y las operaciones con tecnologías inteligentes que se integran en las organizaciones, las personas y los activos. Esta nueva etapa de la industria apuesta por una mayor automatización, conectividad y globalización, mientras que la llegada de las nuevas tecnologías como la robótica, big data, la inteligencia artificial y el internet de las cosas (IoT) ayudan a optimizar los procesos de fabricación, su supervisión e integración con otros procesos y sistemas utilizados.

Palabras Clave: Industria 4.0 (I 4.0), Aplicaciones de I 4.0, Inteligencia artificial, Datos masivos, El internet de las cosas (IoT).

\section{Abstract}

This paper addresses a literary exploration of Industry 4.0 in the most recent years. Industry 4.0 is the current industrial revolution, which consists of the digitization of industrial processes with the help of information technology (IT) and artificial intelligence (AI). This means combining advanced production and operations techniques with smart technologies that are integrated into organizations, people, and assets.This new stage of the industry is committed to greater automation, connectivity, and globalization, while the arrival of new technologies such as robotics, big data, artificial intelligence, and the Internet of Things (IoT) help to optimize manufacturing processes, its supervision and integration with other processes and systems used.

Keywords: Industry 4.0, Applications of I 4.0, Artificial intelligence, big data, the internet of things (IoT). 
10

\section{Introducción}

La producción industrial ha sido moldeada por revoluciones durante siglos. Después de la mecanización (Industria 1.0), la producción en masa (Industria 2.0) y la automatización (Industria 3.0), la llegada de Internet de las cosas y los servicios a la fabricación está dando lugar a la cuarta revolución industrial: Industria 4.0. La Industria 4.0 hace que la producción sea más individual y eficiente (Schawab, 2016). Los conceptos de industria 4.0 y manufactura inteligente, son relativamente nuevos $y$ contemplan la introducción de las tecnologías digitales en la industria de la fabricación. Es decir, la incorporación al ambiente de manufactura de tecnologías como el internet de las cosas, cómputo móvil, la nube, el big data, redes de sensores inalámbricos, sistemas embebidos y dispositivos móviles, entre otros (Kumar, 2015).

Algunas de estas tecnologías, ya han sido utilizadas por años, pero de forma aislada; sin embargo, su integración y posibles capacidades, es lo que las potencializa para transformar la industria de la manufactura, con procesos productivos totalmente integrados, automatizados y optimizados; y con resultados significativos en el mejoramiento de la eficiencia operativa y el desempeño organizacional (Suarez, 2016).

Si bien existen ciertas diferencias en las acepciones para connotar el advenimiento de esta cuarta revolución industrial (Industria 4.0, fabrica inteligente, el Internet de las cosas industrial), existe coincidencia en que se trata de procesos de automatización inteligente de las industrias manufactureras que implica la interconexión de partes sensibles de las empresas de modo que esta mejore su adaptabilidad mediante procesos de inteligencia artificial donde convergen los sistemas ciber físicos a los sistemas de producción industriales (Drath \& Horch, 2014; Strozzi, Colicchia, Creazza, \& Noè, 2017).

\section{Fundamentos teóricos}

\section{Industria 4.0 y manufactura inteligente.}

El fenómeno de la Industria 4.0 se mencionó por primera vez en 2011 en Alemania como una propuesta para el desarrollo de un nuevo concepto de política económica alemana basado en estrategias de alta tecnología (Mosconi, 2015). El concepto ha lanzado la cuarta revolución tecnológica, que se basa en los conceptos y tecnologías que incluyen sistemas ciber físicos, Internet de las cosas (IoT) e Internet de servicios (Andersson P, 2015), basado en la comunicación perpetua a través de Internet que permite una interacción e intercambio continuo de información no solo entre humanos (C2C) y humanos y máquinas (C2M) sino también entre las propias máquinas (M2M; Cooper \& James, 2009). Esta interacción comunicacional influye en el establecimiento de la gestión del conocimiento 4.0 (Roblek, 2016).

\section{Pilares Tecnológicos de la Industria 4.0}

La Industria 4.0 permitirá recopilar y analizar datos en todas las máquinas, lo que permitirá procesos más rápidos, más flexibles y eficientes para producir bienes de mayor calidad a costos reducidos. Esto, a su vez, aumentará la productividad de la fabricación, cambiará la economía, fomentará el crecimiento industrial y modificará el perfil de la fuerza de trabajo, lo que en última instancia cambiará la competitividad de las empresas y las regiones. Sin embargo, ahora estamos en medio de una cuarta ola de avance tecnológico: el surgimiento de la nueva tecnología industrial digital conocida como Industria 4.0., una transformación impulsada por nueve avances tecnológicos fundamentales (BCG, 2015). 
Entre los pilares tecnológicos de la industria 4.0 se encuentran:

Sistemas de Integración: Son sistemas con capacidades físicas y de cómputo integradas, que pueden interactuar con humanos a través de diversos medios; permiten acceder a los datos y servicios disponibles en la web; monitorean y controlan los procesos físicos $y$ hacen las conexiones entre el mundo real y virtual con el internet de los servicios y la fábrica inteligente, así lo argumenta (Ynzunza, Izar, \& Bocarando, 2017).

Máquinas y sistemas autónomos (Robots): En el mundo de la industria, la tendencia es avanzar sobre la automatización de los procesos productivos, el control, la integración de sensores y actuadores, la comunicación de las interfaces. Se busca incrementar la robótica colaborativa para ir hacia fábricas inteligentes donde todas las áreas de la empresa puedan trabajar en forma conectada y con alto nivel de automatización en las tareas (Basco, Beliz, Coatz, \& Garnero, 2018).

Manufactura Aditiva: La manufactura aditiva permite fabricar piezas a partir de la superposición de capas de distintos materiales tomando como referencia un diseño previo, sin moldes, directamente desde un modelo virtual. Esta tecnología descentraliza las etapas de diseño y desarrollo de productos e introduce un mayor componente de servicios y software a las manufacturas (Basco, Beliz, Coatz, \& Garnero, 2018).

El Internet de las Cosas (IoT): El término Internet de las Cosas (IoT) fue acuñado por primera vez por el pionero de la tecnología británica Kevin Ashton en una presentación que realizó en 1999 para la multinacional Procter \& Gamble, donde describía un sistema en el cual los objetos en el mundo físico podrían conectarse a Internet a través de sensores para automatizar la recogida de datos, con aplicación en la cadena de suministro (Ashton, 2018).

El IoT, como se ha adelantado, a una tecnología basada en la conexión de objetos cotidianos a Internet que intercambian, agregan y procesan información sobre su entorno físico para proporcionar servicios de valor añadido a los usuarios finales. También reconoce eventos o cambios, y tales sistemas pueden reaccionar de forma autónoma y adecuada. Su finalidad es, por tanto, brindar una infraestructura que supere la barrera entre los objetos en el mundo físico y su representación en los sistemas de información. También, la integración de sensores y dispositivos en objetos cotidianos que quedan conectados a Internet a través de redes alámbricas e inalámbricas (Andrés, 2018). Puede encontrarse una amplia compilación del estado actual de las arquitecturas de IOT, las tecnologías y las metodologías para su implementación en la publicación de (Ray, 2018), donde incluye además referencias a escenarios de aplicación como: monitoreo de variables ambientales, agricultura, redes de sensores distribuidas, cadenas de suministro, cuidado de la salud, ciudades inteligentes y otros casos.

Big Data y análisis de grandes datos: Big Data es un término común bajo el que se agrupan toda clase de técnicas de tratamiento de grandes volúmenes de datos, fuera de los análisis y herramientas clásicas. A pesar de que el término Big Data se asocia principalmente con cantidades de datos exorbitantes, se debe dejar de lado esta percepción, pues Big Data no va dirigido solo a gran tamaño, sino que abarca tanto volumen como variedad de datos y velocidad de acceso y procesamiento. En la actualidad se ha pasado de la transacción a la interacción, con el propósito de obtener el mejor provecho de la información que se genera minuto a minuto (Mohanty, 2015). 
En este modo, con el auge del Big Data se ha dado cabida también a un nuevo concepto, Data Science o Ciencia de los Datos, que se usa de forma genérica para hacer referencia a la serie de técnicas necesarias para el tratamiento y manipulación de información masiva desde un enfoque estadístico e informático. Incluyendo también el surgimiento de un nuevo perfil profesional, el "Data Scientist" (Mertins, 2014) las personas capacitadas en este perfil deben saber del negocio, de las herramientas computacionales y de análisis e interpretación estadística.

Computación en la nube: La computación en la nube es un modelo que permite el acceso a la red a pedido, conveniente y ubicuo a un grupo compartido de recursos informáticos configurables (por ejemplo, redes, servidores, almacenamiento, aplicaciones y servicios) que se pueden aprovisionar rápidamente. y se publica con un mínimo esfuerzo de gestión o interacción con el proveedor de servicios (National Institute of Standards and Technology, 2015).

Así, el cómputo en la nube es más que una tecnología absoluta o total por sí misma; es decir, se trata más de un modelo de servicios de TIC para el acceso, la asignación, el control y la optimización de los recursos, entendida como una puesta a disposición de recursos para el disfrute del usuario en diversas modalidades de servicio e implementación (Cloud Security Alliance, 2009).

La computación en la nube es más una evolución tecnológica, que un concepto novedoso. Entre las principales evidencias históricas se encuentran; primero, el uso que empresas y universidades hacían de las grandes computadoras centrales, lo que hoy en día se conoce como servicio de accesibilidad remota a los recursos de procesamiento de otra computadora y segundo, la aparición de las redes privadas virtuales VPN (Virtual Private Network) (Liao y Su, 2011), los servicios ofrecidos a través de esta topología de red se extrapolan a los servicios que hoy en día se ofrecen a través de la computación en la nube. Gracias a los servicios de la computación en la nube la tendencia actual está orientada a contratar servicios externos en centros de datos ubicados en cualquier parte del mundo.

Ciberseguridad: La Ciberseguridad Industrial es el conjunto de prácticas, procesos y tecnologías, diseñadas para gestionar el riesgo del ciberespacio derivado del uso, procesamiento, almacenamiento y transmisión de información utilizada en las organizaciones e infraestructuras industriales, utilizando las perspectivas de personas, procesos y tecnología. (Alter Technology, 2019). La ciberseguridad es el aspecto más sensible de la comunicación y los negocios debido al mayor uso de Tecnologías de la Información y las Comunicaciones (TIC), el incremento de la conectividad y la transformación digital de las organizaciones, los gobiernos y la sociedad (Endeavor México, 2020).

En la industria 4.0 existirán momentos críticos en donde la ciberseguridad será una pieza clave para mantener en orden todos los procesos. El primero de ellos es la actualización de software de cada uno de los dispositivos que interactúan entre sí pues con cada nueva actualización, las vulnerabilidades pueden aumentar o disminuir, dependiendo de las propias características de la actualización (Romero, 2018).

Realidad aumentada: La realidad aumentada consiste en la mezcla de contenido digital con contenido físico para construir una realidad mixta en tiempo real. De ahí viene 
su nombre: se incrementa la realidad a través de la incorporación de información adicional y además se ubica en el espacio. (Asociación Cluster de Automoción de Navarra, 2017). Para ello es necesario crear un entorno tridimensional, ya sea ficticio o real y, a través de aplicaciones específicas, visualizarlo en unas gafas de realidad virtual o soportes para dispositivos móviles.

Dicha realidad puede ser pasiva, como en las películas de 3D, exploratoria como las visitas virtuales a un museo o interactiva cuando además de ver, oír y desplazarse permite interactuar con el entorno para lo que es necesario utilizar sensores y mandos. Además, puede ofrecer una inmersión total en el mundo virtual o a través de un monitor. (Fischer, A Cultural Perspective on Mixed, Dual, 2011).

La realidad aumenta y la realidad virtual van de la mano pues están muy relacionadas, aunque la virtual si está más desarrollada en la sociedad, poseen características comunes como la inclusión de modelos virtuales, gráficos $2 \mathrm{D}$ y $3 \mathrm{D}$ para experiencias de usuario; sin embargo, su principal diferencia es que la realidad aumenta no remplaza el mundo real por uno virtual, sino que, por el contrario, mantiene al individuo en el mundo real y lo complementa con información virtual, con la que incluso puede interactuar (X. Basogain, 2007).

Simulación: Permite ajustar y representar virtualmente el funcionamiento conjunto de máquinas, procesos y personas en tiempo real antes de ser puestos en marcha, lo que ayuda a prevenir averías, ahorrar tiempo y evaluar el resultado final en un entorno controlado. Es decir, permite reducir los costos asociados a procesos de aprendizaje (de "prueba y error") mediante una representación virtual para el diseño de nuevos productos, o bien probar distintas configuraciones en las operaciones de la planta productiva. (Ana Inés Basco, 2018). La simulación por ordenador se utiliza actualmente en numerosas disciplinas: educación, ciencia y tecnología, economía, ciencias sociales, medicina, ingeniería, arquitectura, industria, construcción.

En la fase de ingeniería, ya se utilizan simulaciones 3-D de productos, materiales y procesos de producción, pero en el futuro, las simulaciones también se utilizarán de manera más extensa en las operaciones de la planta. Estas simulaciones aprovecharán los datos en tiempo real para reflejar el mundo físico en un modelo virtual, que puede incluir máquinas, productos y humanos. Esto permite a los operadores probar y optimizar la configuración de la máquina para el siguiente producto en línea en el mundo virtual antes del cambio físico, lo que reduce los tiempos de configuración de la máquina y aumenta la calidad. (Markus Lorenz, 2015)

Por ejemplo, Siemens y un proveedor alemán de máquinas herramienta desarrollaron una máquina virtual que puede simular el mecanizado de piezas utilizando datos de la máquina física. Esto reduce el tiempo de preparación para el proceso de mecanizado real hasta en un 80 por ciento (Digital, 2019).

Campo de aplicación de la industria 4.0 en diferentes sectores

\section{Sector energético}

El sector energético es otro de los beneficiados de las tecnologías de la industria 4.0. El tratamiento de grandes cantidades de datos, conocido como Big Data, permite optimizar el uso de energía, determinar los tipos de energía se disponen en cada momento a menor costo e incluso 
14

se prevé que toda esta innovación permita mejorar y desarrollar un mejor uso de los combustibles fósiles y biocombustibles (Atria Innovation, 2019).

Los cambios que se avecinan en empresas, industrias y redes locales se engloban en un nuevo escenario compuesto por ciudades inteligentes $y$ el uso extensivo del vehículo eléctrico, la existencia de un fuerte compromiso con la eficiencia energética, la descarbonización, la sostenibilidad y el medio ambiente (Carlos Rivas Pereda, 2017). Gracias a las aplicaciones de la industria 4.0, ya no es algo futurista el que los procesos en las plantas estén interconectados, o la visión a tiempo real de la operabilidad y estado de todas las máquinas que conforman los procesos de producción. De esta forma, la industria 4.0 permite la gestión de recursos para lograr una mayor eficiencia, producción flexible e integración de procedimientos globales a través de un software en el uso de la energía durante los procesos productivos (Cosio, 2019).

En este marco, la mayor revolución y a la vez principal necesidad del sector energético es incorporarse a esta industria y conectar todos los nuevos sistemas con los ya existentes, y alcanzar la plena digitalización, aunque palabras como internet de las cosas, blockchain e inteligencia artificial ya se mencionan en el sector.

\section{Sector Salud}

La industria 4.0 está siendo empleada para múltiples usos relacionados con el área de la salud, buscando generar nuevos métodos para la detección, prevención, tratamiento y diagnóstico de enfermedades, garantizando a las personas una mejor calidad de vida, bienestar, una mejor atención médica, más exacta y oportuna que le permita a los pacientes actuar de manera rápida y efectiva (Mohamed Elhoseny, 2017).

A su vez se propone un sistema de intercambio de datos médicos que permita el acceso y colaboración entre varias entidades prestadoras del servicio de salud. Esto, con el fin de maximizar la utilización de recursos y facilitar la ejecución de tareas tales como la atención médica.

En el mismo orden de ideas (Leila Ismail, 2020). Principalmente a través de la tecnología "Blockchain" y junto con otras como la de Big Data, propone un modelo para la optimización de actividades en los centros médicos tales como el diagnóstico de enfermedades, recopilar información de los pacientes y mejorar la accesibilidad de datos entre proveedores de atención médica.

\section{Sector económico}

El desarrollo experimentado por las tecnologías de la información y de la comunicación (TIC) o tecnologías digitales en las últimas décadas y las innovaciones empresariales asociadas a ellas, han definido un espacio económico. (Cohen, Stephen, J. Bradford De Long y John, 2000). Por otra parte, según (Rob Kling, Roberta Lamb, 1999) la economía digital es un sector de la economía que incluye los bienes y servicios en los que su desarrollo, producción, venta o aprovisionamiento dependen de forma crítica de las tecnologías digitales. La evolución hacia la economía 4.0 requiere un cambio cultural, debe ir acompañada de gestión del cambio, para asegurar que haya una adopción correcta de los sistemas. En las industrias 4.0, la transformación de los procesos involucra una mayor integración entre comercialización, producción y distribución (fuentes, 2019).

Sector industrial 
En el ámbito de la industria, se pone de manifiesto la potencialidad de la digitalización para la modernización del proceso de producción y la transformación de la actividad industrial. Las tecnologías digitales ofrecen nuevas oportunidades de mejora en los procesos productivos, aumentan la productividad, la eficiencia energética, la eficiencia en el uso de los recursos y la eficiencia de costes, contribuyendo, al mismo tiempo, a mejorar la competitividad de las empresas, lo que redunda en un incremento del bienestar para los empleados y una reducción del impacto ambiental (Confederación Sindical de Comisiones Obreras, 2017).

La automatización de la producción definida como la tecnología referida a la aplicación de sistemas basados en computación, mecánicos y electrónicos para la operación y el control de la producción (Serna, 2017). Por tanto, es factible que en el futuro todos estos cambios tendrán un fuerte impacto en el contenido del trabajo, en la manera en que éste se lleva a cabo; y de igual modo reconfiguren la forma en la que las personas forman parte y agregan valor a las organizaciones (Thomas Bauernhansl, Michael ten Hompel, Birgit Vogel Heuser, 2014). Por otra parte, se sabe que habrá consecuencias tanto en la manufactura como en los recursos humanos, no solo en aquellos con menores competencias, sino también alcanzará a aquellos con mayores niveles de estudio (Linda Bonekamp, 2016).

\section{El recurso humano en la industria $\mathbf{4 . 0}$}

La adopción de tecnologías de manufactura avanzada dentro de los procesos industriales y productos da paso a la fábrica del futuro. A pesar de todos los beneficios de la Industria 4.0, muchas empresas enfrentan grandes obstáculos para aprovechar plenamente las ventajas de la fabricación digital: puede ser extremadamente difícil para las organizaciones integrar a su gente a estos procesos altamente orientados por datos y automatizados.
Aunque las innovaciones tecnológicas están en el centro de lo que hace la Industria 4.0 tan revolucionaria, personas siguen siendo un componente clave del éxito en los negocios de cualquier empresa. Organizaciones que han adoptado o están pensando en adoptar la industria 4.0 deben considerar siempre el factor humano en sus operaciones (Solutions, 2021).

\section{Perspectivas futuras y beneficios de la industria 4.0}

\section{Conceptualización y componentes de la industria 4.0}

LaIndustria 4.0, hace referencia a tecnologías y conceptos de la organización de la cadena de valor en fábricas inteligentes, que cuentan en su estructura con sistemas ciber físicos (CPS) capaces de monitorear los procesos físicos, crear una copia virtual del mundo real y hacer decisiones descentralizadas. Por lo cual, se espera que los CPS brinden soluciones que permitan transformar la operación y el papel de muchos de los sistemas industriales existentes (Varghese y Tandur, 2014). Por lo que, el IoT y la industria 4.0 incidirán también en la forma en la que interactúan los clientes, proveedores y mayoristas, etc., quienes podrán tener una mayor participación en el proceso y las decisiones acerca de la manufactura, calidad y personalización de los productos; considerando por supuesto para ello, los desafíos de ciberseguridad implicados que garanticen el contar con una estructura sólida de intercambio de información y colaboración (Ching-Sung Wang, 2011). 
Tabla 1. Conceptualización de los componentes principales de la industria 4.0

\begin{tabular}{cl}
\hline Sistemas ciber físicos (CPS) & $\begin{array}{l}\text { Los sistemas ciber físicos es un proceso que integra la computación con los procesos físicos. Los } \\
\text { computadores embebidos, el monitoreo de redes y el control de procesos físicos, usualmente tienen } \\
\text { ciclos de retroalimentación en los que los procesos físicos afectan los cálculos y viceversa (Chandy, } \\
\text { 2010). }\end{array}$ \\
\hline Internet de las cosas (LoT) & $\begin{array}{l}\text { El Internet de las cosas (IoT) percibe un mundo donde los dispositivos que lo conforman pueden ser } \\
\text { identificados en el Internet y está creciendo a un ritmo acelerado con nuevos dispositivos que se van } \\
\text { conectando (Cama Pinto, Alejandro De la Hoz, Emiro, 2012). }\end{array}$ \\
\hline Internet de los servicios (LoS) & $\begin{array}{l}\text { Infraestructura para la distribución de las actividades de valor agregado, modelos de negocio y } \\
\text { servicios que se ofrecen a través de internet (Giusto, D., Iera, A., Morabito, G., Atzori, L, 2010). }\end{array}$ \\
\hline Maquinas inteligentes & $\begin{array}{l}\text { Las máquinas inteligentes poseen dentro de sus elementos programas o software que se ejecutan } \\
\text { internamente en su circuitería, y que tienen la capacidad de imitar algunas de las acciones de un ser } \\
\text { humano, procurando igualarlas e incluso excederlas; es decir, intentando emular una parte o la } \\
\text { totalidad de la inteligencia humana (Gonzales, 2017) }\end{array}$ \\
\hline $\begin{array}{c}\text { Los productos inteligentes y conectados son productos, activos y otros objetos que cuentan con } \\
\text { procesadores, sensores, software y conectividad que permite el intercambio de datos entre el producto } \\
\text { y su ambiente, fabricante, operador/usuario, y otros productos y sistemas (Guirola, 2018) }\end{array}$ \\
\hline Productos inteligentes
\end{tabular}

Fuente: Elaboración propia en base los conceptos de diferentes autores

\section{Perspectivas futuras}

La Industria 4.0 es una realidad, y el enfoque continúa evolucionando a medida que el proceso madura, sin embargo, los conceptos determinantes son: Los sensores, pues estarán involucrados en todas las etapas del proceso de fabricación, proporcionando los datos en bruto, así como la retroalimentación que es requerido por los sistemas de control (Sendler, 2013).

Las tecnologías digitales brindan a la industria manufacturera la posibilidad de dar respuesta a las nuevas exigencias de sus clientes a nivel de Productos, Procesos y Modelos de negocio, produciendo un impacto determinante en la cadena de valor (Blanco, 2016).

Algo relevante de la Industria 4.0 es que no solo es la nueva perspectiva de la producción industrial, productos y soluciones, sino que tiene la obligación de mantener niveles de estándar y seguridad informática, de datos y procesos durante la fabricación de los productos (Tapia, 2014).

La Tabla 2, detalla la arquitectura tecnológica y de soporte que forma parte de la industria 4.0 y la manufactura inteligente, así como algunos de los beneficios que han sido vinculados a éstas. En la misma, se precisan los artefactos tecnológicos integrados, las capacidades que permiten los mismos, dadas sus características y principios de diseño, así como el detalle de la arquitectura de soporte y los beneficios asociados.

\section{Beneficios}

Al introducir este nuevo método se esperan resultados favorables que desencadenen en una alta competitividad de la industria con respecto a las demás del mercado, ya que ofrece muchos beneficios a través de la introducción de la digitalización (Lean Manufacturing 10, 2019). 
Tabla 2. Perspectivas y beneficios de la industria 4.0

\begin{tabular}{|c|c|c|c|}
\hline $\begin{array}{c}\text { Fabricas } \\
\text { inteligentes }\end{array}$ & $\begin{array}{c}\text { Ciudades } \\
\text { inteligentes }\end{array}$ & $\begin{array}{c}\text { Productos } \\
\text { inteligentes }\end{array}$ & $\begin{array}{c}\text { Servicios } \\
\text { inteligentes }\end{array}$ \\
\hline \multicolumn{3}{c}{ Artefactos tecnológicos integrados } \\
\hline \\
Sensores, Sistemas autónomos, Microchips, Sistemas ciber físicos, Maquinas \\
inteligentes
\end{tabular}

\section{Características}

Flexibilidad, Inteligencia, Conectividad, Seguridad, Confiabilidad, Trazabilidad, Movilidad, Sustentabilidad

\begin{tabular}{l} 
Principios de diseño \\
\hline $\begin{array}{l}\text { Integración, Interoperabilidad, Capacidad en tiempo real Descentralización, } \\
\text { virtualización, Orientación al servicio, Modularidad. }\end{array}$
\end{tabular}

\section{Arquitectura del soporte}

Internet de las cosas (LoT), Big data, Redes industriales, DaaS (Datos como servicio), PaaS (La plataforma como servicio), Maas (La monitorización como un servicio), Computo en la nube.

\section{Beneficios}

Mayor productividad y una mejor gestión de los recursos, Toma de decisiones más eficiente basada en información real, Procesos productivos óptimos e integrados, Aumento de la flexibilidad, Comunicación directa entre los clientes y las organizaciones, Reducción de tiempo. Reducción del porcentaje de defectos.

Fuente: Elaboración propia en base a: (Carmen Ynzunza, Juan Izar, Jacqueline Bocarando, Felipe Aguilar, Martín Larios, 2017)

\section{Retos de la industria 4.0}

La adaptación hacia el nuevo modelo industrial debe comenzar por considerar la digitalización como una oportunidad para adaptar o crear nuevos procesos, productos y modelos de negocio.

En lo que a talento respecta, la clave es innovar para agregar valor a los trabajos y procesos. De esta forma, existirán mayores probabilidades de mantener y mejorar el nivel competitivo internacional. Asimismo, como se menciona en el reporte Workforce of the Future, es importante invertir en la educación y la readaptación de procesos empresariales y de recursos humanos (Carlos Zegarra, Moisés Pérez, 2018).
Los retos, por tanto, que acompañan el despliegue de la Industria 4.0 son numerosos, pero si analizamos con detenimiento el acelerado progreso tecnológico en curso, y los efectos que plantean las actuales innovaciones disruptivas en la industria, podríamos destacar cuatro retos como los principales, en el proceso de digitalización: i) el necesario cambio de la cultura empresarial de las organizaciones industriales que debe acompañar a la transformación digital; ii) la formación y capacitación en competencias digitales; iii) el desarrollo de La industria conectada 4.0 y iv) el apoyo a todas las empresas, especialmente pequeñas y medianas, en su proceso de transformación digital (Mario Buisán, Fernando Valdés, 2017). 
Tendencias actuales de la industria 4.0

Tabla 3. Retos e Implicaciones de la industria 4.0

Automatización industrial, Robótica colaborativa, Robots que asisten e interactúan con humanos, Máquinas que aprenden, que procesan las experiencias pasadas para ser autodidactas, Productos inteligentes, conscientes del entorno y que proveen información, Manejo y análisis de grandes volúmenes de datos en tiempo real, Enfoque a la innovación y actividades de valor agregado.

\section{Negocios}

Cambios en el tipo de productos de físico a digitales conectados, Mayores niveles de integración de la organización y el entorno, Redes de trabajo que crean valor, Infraestructura y servicios compartidos en la nube, Cadenas de suministro inteligentes y globales, Modelos de negocio basados en servicios en la nube, logística y distribución.

\section{Empleo}

Nuevos esquemas de colaboración e infraestructuras sociales, Otras formas de interacción hombre-máquina, Perfiles de puesto altamente especializados., Procesos de trabajo más complejos, Trabajo más flexible, Ambientes de trabajo asistidos y dependientes de la tecnología., Requerimientos para el manejo de tecnologías digitales, robots, programación y analíica como competencias transversales.

Fuente: Elaboración propia a partir de :(Cooper \& James, 2009; Christopher \& Holweg, 2011; Bloom \& Sadun, 2012; Lasi, Fettke, Feld, \& Hoffmann, 2014; Roblek, Meško, \& Krapež, 2016).

\section{Conclusiones}

Este artículo abordo una revisión literaria de la industria 4.0 y las últimas tendencias, así como los principales pilares que la conforman, el campo de aplicación y perspectivas futuras.

La industria 4.0 es la digitalización de los procesos productivos mediante sensores y sistemas de información que los hacen más eficientes, gracias a la integración de este tipo de tecnologías y la automatización de la manufactura se puede disponer de los datos generados por estos sistemas en tiempo real, procesarlos para tomar decisiones con ayuda de herramientas de analítica avanzada y tomar la mejor decisión que ayude a optimizar los procesos tanto productivos, económicos y comerciales que se encuentren dentro de la cadena de valor de la empresa o producto a la cual se adapte.

Los retos e implicaciones que aborda la industria 4.0 es la automatización industrial, los sistemas de análisis masivos de datos que implica el almacenamiento y análisis de estos, la incorporación de robots que interactúen con los humanos, la incorporación de elementos electrónicos que interactúen con los procesos productivos, el cambio del entorno de la organización y nuevos esquemas de trabajo. Por otra parte, uno de los retos más grandes es la incorporación del factor humano en la industria 4.0 debido que es difícil para las organizaciones integrar a su gente a estos procesos altamente orientados por datos y automatizados, si bien podría ser una amenaza, es una gran oportunidad para nuevos puestos de trabajo, mano de obra más especializada, trabajadores multidisciplinarios y mejor pagados.

Por otra parte, la industria 4.0 se orienta hacia un futuro altamente conectado y es por ello que las industrias, las pequeñas y medianas empresas se están enfocando en la adaptación de este tipo de tecnologías ya que los beneficios son mayores y este cambio en algún momento alcanzara a mayoría de las empresas en el sector industrial, lo cual orilla a las industrias capacitarse y desarrollar nuevos productos que se adapten a las necesidades que implica la industria 4.0 y así aprovechar las ventajas que ofrece la industria conectada.

La tecnología evoluciona cada vez más rápido y las empresas deben adaptarse a los cambios lo antes posible para seguir siendo una ventaja competitiva en el mercado. La digitalización de la industria es una revolución que está cambiando la forma en la que se 
realizan los procesos industriales, los entornos de trabajo y la forma de relacionarse tanto con proveedores como con clientes dentro de la misma cadena de suministro.

Sabemos que esto apenas es el comienzo del camino hacia un futuro innovador, no solo dentro del sector industrial, sino también dentro de otros sectores que se han adaptado al uso de estas nuevas tecnologías para el bienestar social y mejorar la calidad de vida.

\section{Referencias}

Albrieu, Ramiro; Basco, Ana Inés; Brest López, Caterina; De Azevedo, Belisario; Peirano, Fernando; Rapetti, Martín; Vienni, (2019). Travesía 4.0: Hacia la transformación industrial argentina,12-15

AMETIC (2020). Ametic y su compromiso con la industria 4.0. Documento recuperado de:https://ametic.es/sites/default/files// presentacion_industria40.pdf

Andersson, P. and Mattsson, L.-G. (2015), "Service innovations enabled by the "internet of things"", IMP Journal, 9(1), 85-106. Doi. https://doi.org/10.1108/IMP-01-2015-0002

Asociación Cluster de Automoción de Navarra. (2017). La industria 4.0 tecnologías habilitadas. Recuperado de https:// clusterautomocionnavarra.com/wp-content/ uploads/2017/10/ACAN

Ashton, K. (2018). Tecnólogo experto en Transformación Digital y creador del concepto el Internet de las Cosas. Texto publicado en Executive Excellence $\mathrm{n}^{\mathrm{0}} 150$ julio-agosto 2018. http://www.eexcellence.es/ index.php/expertos-en-gestion/kevin-ashtonun-tecnologo-visionario

Basco, A.; Beliz, G.; Coatz, D.; Garnero, P.
(2018). Industria 4.0: fabricando el futuro. Banco Interamericano de Desarrollo. Recuperado de: https://publications.iadb.org/ publications/spanish/document/Industria-40Fabricandoel-Futuro.pdf

Basogain, X., Olabe, M., Espinosa, K., Rouèche, C. y Olabe, J. (2007). Realidad aumentada en la edu-cación: una tecnología emergente. Madrid: Onli-ne Educa Madrid

Bauernhansl, T. (2014). Die vierte industrielle Revolution-Der Weg in ein wetshaffendes Produktionsparadigma. In $\mathrm{T}$ Bauernhansl, M. Ten Hompel \& B. Vogel-Heuser (Eds). Industrie 4.0 in Produktion, Automatisierung und Logistik Anwendungen Technologies Migration Wiesbaden: Springer, 5-36

Bloom, N., \& Sadun, R. \&. (2012). Americans do I.T. better: US multinationals and the productivity miracle. American Economic Review, 102, 167-201

Buisán, M., Valdés F. (2017), La industria conectada 4.0, la economía digital en españa

Cama Pinto, A., De la Hoz Franco, E., \& Cama Pinto, D. (2012). Las redes de sensores inalámbricos y el internet de las cosas. INGE CUC, 8(1), 163-172. Recuperado a partir de https://revistascientificas.cuc.edu.co/ingecuc/ article/view/253

Centro de Ciberseguridad Industrial CCI. Recuperado de: https://www.cci-es.org/

Chandy, J. C. (2010). Desafíos en el diseño de sistemas CiberFísicos. Ingenierías USBMed,(1), 6-14

Cosio, M. (2019). Digitalización, el nuevo aliado del sector energético. Recuperado a partir de:https://e-management.mx/digitalizacionel-nuevo-aliado-del-sector-energetico/ 


\section{0}

Cohen, Stephen, J. Bradford DeLong y John Zysman (2000). Tools for Thought. Berkeley: University of California.

Cooper, J., James, A. ( 2009 ). Retos para la gestión de bases de datos en Internet de las cosas . Revisión técnica del IETE, 26, 320 $-329$

Confederación Sindical de Comisiones Obreras, 2017. La Digitalización y la Industria 4.0. Recuperado de: h https:// c51a3697f785ba14fce86528e10000060.pdf

De Fuenmayor, A. (2017), Cloud computing, ¿el final de una era?. Recuperado de:https:// empresas.blogthinkbig.com/cloudcomputing-iot/

Duncan McFarlane, Mudassar Ahmed 2017, Selecting Additive or Dedicated Manufacturing: A Strategic Technology Choice, Institute for Manufacturing (IfM), Cambridge University Engineering Recuperado de https:// connectedeverythingmedia.files.wordpress. com/2018/06/ce-isda2018.pdf

DuPont Sustainable Solutions (2021). No pierda de vista el "factor humano" en la industria 4.0, Recueprado de: https://www.latam. dsslearning.com/content/pdf/white-papers/ factor-humano-en-la-industria-spn.pdf

Elhoseny, M., Abdelaziz, A., Salama, S., Riad, M., Muhammad, K. and Sangaiah, A.K. (2018). "A hybrid model of Internet of Things and cloud computing to manage big data in health services applications,'Futur. Gener. Comput. Syst., vol. 86, pp. 1383-1394

Endeavor México, Panorama del ecosistema de ciberseguridad 2020. Disponible en https://www.endeavor.org.mx/articulos data_lab/Panorama-del-ecosistema-deciberseguridad/Panorama-del-ecosistema- de-ciberseguridad.pdf

Fischer, M.,, Sally, A. (2011). Cultural Perspective on Mixed, Dual and Blended Reality 1-4

Fuentes 2019 , Econoomia 4.0, Recuperado de: https://www.grupoabsa.com/blog/blog-1/ post/economia-4-0-15

Giusto, D., Iera, A., Morabito, G., Atzori, L. (2010). The Internet of Things. SpringerVerlag New York

Gonzales 2017, Smart machines, Investiga TEC Recuperado a partir de: https://revistas. tec.ac.cr/index.php/investiga_tec/article/ download/3142/2862/8569

Guía para la Seguridad en áreas Críticas de atención en Cloud computing V.2, traducida por la Asociación Española para el Fomento de la Seguridad de la Información en 2009. Recuperado de: https://cloudsecurityalliance. org/guidance/csaguide-es.v2.pdf (consultada en octubre de 2011)

Lee, J., Davari, H., Singh, J., Pandhare, V. (2018). "Industrial Artificial Intelligence for industry 4.0-based manufacturing systems," Manuf. Lett., vol. 18, pp. 20-23, Doi: 10.1016/j. mfglet.2018.09.002

Lasi, H., Fettke, P., Feld, T., \& Hoffmann, M. (2014). Industry 4.0. Business \& Information Systems Engineering, 6 (4), 239-242.

Lean Manufacturing10 (2019), Recuperado de: https://leanmanufacturing10.com/ industria-4-0

Lorenz, M., Rüßmann, M., Waldner,M. (2015). Pascal Engel, Michael Harnisch, and Jan Justus "Industry 4.0: The Future of Productivity and Growth in Manufacturing

Industries". Recuperado de: https://www. bcg.com/publications/2015/engineered_ 
products_project_business_industry_4 future_productivity_growth_manufacturing industries\#chapter2

Mohanty, H., Bhuyan, P. and Chenthati, D. (2015). Big Data: A Primer, vol. 11. Springer

Montiel Romero, J. (2018). La Ciberseguridad en la industria 4.0. Recuperado de: https:// profesionistas.org.mx/la-ciberseguridad-en-laindustria-4-0/

Mosconi, F. (2015). The new European industrial policy: Global competitiveness and the manufacturing renaissance. London, England: Routledge

Ning, H., \& Liu, H. (2015). Cyber-physicalsocialthinking space based science and technology framework for the Internet of things

Ray, P.P. (2016). A survey on Internet of Things architectures,'Journal of King Saud University -Computer and Information Sciences, 30 (3), 291-319. Doi: 10.1016/j.jksuci.2016.10.003

Rivas Pereda, C. (2017). Energía 4.0 Tecnologías innovadoras en el sector energético. Revista Dinamo Tecnica, 21, 21-23

Salimbeni, S. (2020). Coronavirus: la transformación digital en los hospitales. Recuperado de:https://n.com.ar/opinion/ coronavirus-la-transformacion-digital-en-loshospitales_1055316/

Science China Information Sciences, 58, 1-19. Ningenia (2016). Qué es la Industria 4.0. Recuperado de: http://www.ningenia. com/2016/05/31/que-esla-industria-4-0/.

Sendler U. (2013) Industria 4.0 - Dominio de la complejidad industrial con SysLM (Systems Lifecycle Management). En: Sendler U. (eds) Industria 4.0. Xpert.press. Springer
Vieweg, Berlín, Heidelberg. Doi. https://doi. org/10.1007/978-3-642-36917-9_1

Sommer, L. (2015). Industrial revolutionIndustry 4.0: Are German manufacturing SMEs the first victims of this revolution? Journal of Industrial Engineering and Management, 8, 1512-1532

Strozzi, F., Colicchia, C., Creazza, A., \& Noè, C. (2017). Literature review on the 'Smart Factory' concept using bibliometric tools. International Journal of of Production Research, 55(22), 6572-6591

Tanwar, S., Parekh, K. and Evans, R. (2020). Blockchain-based electronic healthcare record system for healthcare 4.0 applications,

Tapia, V. (2014). Industria 4.0 - Internet de las Cosas, Recuperadp de: http://investigacion.utc. edu.ec/revistasutc/index.php/utciencia/article/ download/6/7

The NIST Definition of Cloud Computing,2015 Recommendations of the National Institute of Standards and Technology. Recuperado de http://csrc.nist.gob/publications/ nistpubs/800-145/SP800-145.pdf

Van der Aalst, W. M. P. (2014). "Data Scientist: The Engineer of the Future," in Enterprise Interoperability VI, no. 7, Eds. Springer International Publishing, 13-26

Varghese, A. y Tandur, D. (2014). Wireless requierements and challenges in industry 4.0. Internatonal Conference on Contemporary Computing and Informatics (IC31), IEEE, 634-638

Vijaykumar, S, Saravanakumar, S, y Balamurugan, M. (2015). Unique sense: smart computing prototype for industry 4.0 revolution with IOT and bigdata implementation model. Indian $\mathrm{J}$. 
Tendencias actuales de la industria 4.0

22

SCi Technol. 8 (35), 1-4

$\mathrm{Xu}, \mathrm{L}$. (2011). Enterprise system: state of art and future trends.. Inf. IEEE Trans. Ind, 7 (4),630640

Ynzunza, C.; Izar, J.; Bocarando, J. Aguilar, F, Larios, M (2017). El Entorno de la Industria 4.0: Implicaciones y Perspectivas Futuras. Revista Conciencia Tecnológica, núm. 54, 2017 Documento en línea. Recuperado de: https:// www.redalyc.org/jatsRepo/944/94454631006/ html/index.html.

Zegarra, C., Pérez, M. (2018), Industria 4.0: oportunidades y retos en México. Recuperado de https://www.forbes.com.mx/industria-4-0oportunidades-y-retos-en-mexico/ 